\title{
Effect of geometry of real-size transparent nozzles on cavitation and on the atomizing jet in the near field
}

\author{
Y. Cao ${ }^{1}$, S. Idlahcen ${ }^{1}$, J.-B. Blaisot*1, C. Rozé ${ }^{1}$, L. Méès ${ }^{2}$, D. Maligne ${ }^{3}$ \\ ${ }^{1}$ CORIA UMR-6614, Université de Rouen, 76801 Saint-Etienne-du-Rouvray, France \\ ${ }^{2}$ LMFA UMR-5509, CNRS, École Centrale de Lyon, 69134 Écully, France \\ ${ }^{3}$ DELPHI France, 41000 Blois, France \\ *Corresponding author: blaisot@coria.fr
}

\begin{abstract}
Cavitation inception is very sensitive to the nozzle geometry. Two diesel-like transparent nozzles with sharp and round inlet edge were investigated under moderate injection pressure. By means of backlight imaging technique, images were recorded in two regions near the orifice: within ten orifice diameter downstream the nozzle and inside the nozzle, for different times after start of the injection. A cross-correlation technique was applied to images downstream the orifice to estimate the average velocity of the spray. The development of the spray was studied at the transient and full-opened stage, related to the needle lift motion. To analyse the flow inside the nozzle, the relative size of the vapour pockets was measured on the image and the relative frequency of the occurrence of cavitation was determined over image series. This approach permits to examine the time variation of the cavitation occurrence from the inlet to the outlet of the orifice. The results highlight the sensitivity of the cavitating flow to the shape of the inlet edge. Moreover, the trends of the variation over time of the velocity and of the cavitation frequency are very similar to each other but different for each geometry, indicating that the characteristics of the diesel spray in the near field is closely affected by the way cavitation is developing in the nozzle.
\end{abstract}

\section{Keywords}

diesel injection, index matching, backlight, liquid interface tracking, frequency of cavitation

\section{Introduction}

Fuel consumption and engine emission are important issues concerning transportation industries. Engine optimization still needs to be improved, not only to balance the global reduction of fossil fuel reserves, but also to reduce their environmental impact. There is a pressing need to address clean combustion objectives, by developing a better understanding and control of every process steps of internal combustion engines. Atomization of fuel is a key element as it impacts the whole combustion process by affecting the initial conditions of this process.

In modern diesel engines, atomization efficiency was improved by considering high injection pressure in combination with small orifice diameter of only about a hundred micrometres or less. Large pressure variations of the fuel flow occurring inside injector nozzles promote cavitation occurrence. Indeed, cavitation occurs when the relative pressure drops locally below the vapour pressure of the fuel or when local stress is very high, resulting in the formation of vapour pockets or cavities in the nozzle orifice [1].

Nurick [2] proposed the first theoretical model to predict nozzle flow under cavitation, based on two-dimensional transparent single-orifice nozzles. Since then, similar nozzle designs were used to analyze the development of the cavitating flow and improve the model, by means of experiments $[3,4,5,6,7]$ and simulations $[8,9]$. The flows in the nozzle were compared by changing either the $L / D$ ratio or the inlet geometry of the orifice. Cavitation inception was found to be very sensitive to the nozzle geometry. Prasetya et al. [6] claimed that the cavitation thickness and the resulting contraction coefficient depend on the inlet edge radius.

For a real-size nozzle, the inlet geometry affects the spray development as well. By comparing cylindrical and conical nozzles, Payri et al. $[10,11,12]$ pointed out that the conicity of the orifice, representing the difference between inlet and outlet diameter, had an impact on the spray formation, specially in the near-field downstream the orifice. Furthermore, in terms of the manufacturing process, the machining precision of the rounding operation at the inlet edge is facing the high sensitivity of cavitation to this geometry. Indeed, a recent analysis of the internal structure of two similar diesel nozzles with a synchrotron source was reported by Wu et al. [13]. A significant difference in inlet edge radius was found even if the design dimensions were the same.

Non-intrusive optical diagnostics are able to provide the velocity information both for the flow inside the nozzle $[4,14,15,16]$ and for the downstream spray $[17,18]$. In their work, Moon et al. [18] argued that the sharp inlet edge induced a higher velocity gradient than the round inlet edge in the transverse direction. In this paper, we use the velocity measurement developed at CORIA $[19,20]$ to investigate the velocity field in the near field of the orifice. This work mainly focuses on the effects of the nozzle geometry, by studying cavitation inception in diesel-like orifices of different shapes, and the effect cavitation on the velocity of a diesel spray in the near nozzle region.

\section{Injector and transparent nozzle}

Injection tests have been conducted with a prototype injector based on a modified DELPHI solenoid Diesel injector. To visualize the flow inside the discharging orifice, transparent nozzles (PMMA) are coupled to the end of the 
stainless nozzle part of the injector body. Two different transparent nozzles have been used in the experiments whose geometry includes a sac and the discharging orifice of diameter $D=0.35 \mathrm{~mm}$ as shown in figure 1 . The main geometry is identically asymmetrical for the two nozzles, with the orifice axis shifted relatively to the sac axis in the side view. As shown after, the asymmetry induces non symmetry in the development of the cavitation inside the orifice, that makes it easier to characterize. In the perpendicular direction (front view), the nozzles are symmetric. The difference between the geometries lies in the connection between the sac and the orifice (see detail in 1), one being sharp and referred to as Sharp edge and the other one being smooth and referred to as Round edge. To visualize cavitation in the transparent nozzles, a refractive index matching method was used by adding high refractive index liquid to calibration oil (ISO 4113) [21]. At ambient temperature $\left(T=20^{\circ} \mathrm{C}\right)$, the vapor pressure of the mixed liquid was found to be around $P_{v}=5 \mathrm{~Pa}$ in a previous study [22].

The current activation signal delivered to the injector by an injector driving system IPOD from EFS company is shown in figure 2. The injection duration is fixed to $T_{i n j}=4 \mathrm{~ms}$ and the injection pressure to $P_{i n j}=30 \mathrm{MPa}$ for both nozzles. The back pressure is kept at ambient value, i.e. $P_{b}=0.1 \mathrm{MPa}$.

The dynamic pressure $P_{\text {tube }}$ is monitored by a pressure sensor mounted on a straight stainless steel tube upstream the fuel inlet of the injector (red curves in figure 2). To characterize the cavitation regime, the cavitation number is defined as $\sigma=\frac{P_{l}-P_{v}}{P_{l}-P_{b}}$ in [2,10], whereas $P_{l}$ is the fuel pressure. To highlight the difference in sac geometry, the pressure in the sac need to be considered in this computation: $P_{l}=P_{s a c}$. However, this measurement is not available in this experiment. Thus, by considering $P_{l}=P_{i n j}$, it leads to an identical cavitation number for both geometries: $\sigma($ Sharp $)=\sigma($ Round $)=1.003$, indicating that cavitation is likely to occur [10, 23]

The injector is equipped with a needle lift sensor (micro-epsilon LS04(04)). The needle lift signal (blue curves in figure 2) presents three parts as usual, i.e. an increase of the needle lift from closed position, a maximum that can have a plateau and a decrease at needle closing. The plateau is observed when the fully opened stage is reached. Here, the plateau is observed at a delay after start of activation ( $A S O A$ ) about $2.2 \mathrm{~ms}$ with the Sharp inlet edge and about $3 \mathrm{~ms}$ with the Round inlet edge. This means that under the same pressure conditions, the Round inlet edge leads to a lower dynamics of the injector needle lift as the time to reach the fully opened stage is increased by almost $50 \%$. It is thus reasonable to assume that the pressure in the sac is significantly different for the two nozzle shapes as this pressure directly controls the needle lift motion speed.

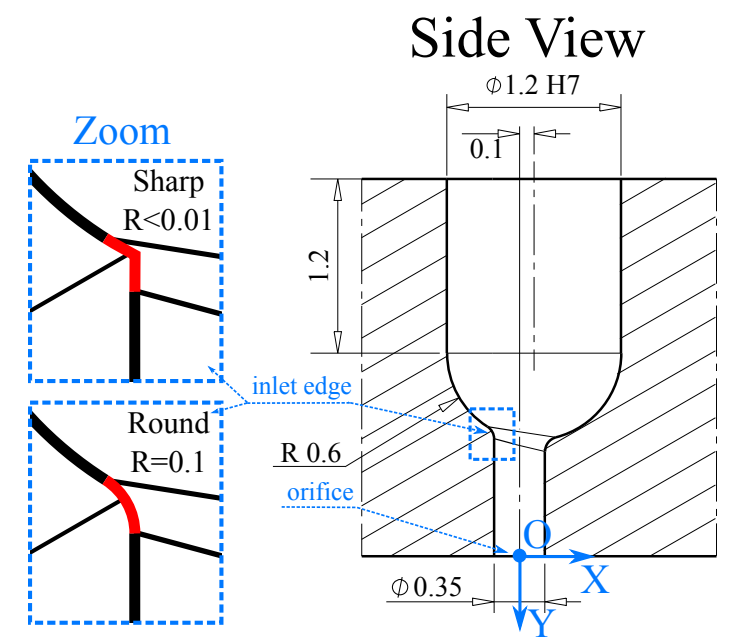

Figure 1. Geometric difference between two nozzle designs. The unit of length is millimetre.

\begin{abstract}
Velocity measurements at fully opened stage
A backlight imaging technique is used to visualize the dynamics of the flow in the nozzle and in the spray, within a distance of about ten nozzle diameters downstream the nozzle orifice. The velocity fields for the Sharp inlet edge were obtained in a previous work [21]. A similar optical setup has been used in the present work for the Round inlet edge. The pulsed light source is a laser diode (Cavitar) with a pulse duration of 10ns (in place of a double cavity femtosecond laser source in [21]). A double frame camera is set to record image-pairs separated by a delay of about $d t=200 \mathrm{~ns}$. The image size is $2048 \mathrm{pixel} \times 2048 \mathrm{pixel}$ and the spatial resolution is about $800 \mathrm{pixel} / \mathrm{mm}$ corresponding to a field of view of $2.58 \mathrm{~mm} \times 2.58 \mathrm{~mm}$ for both views. One should notice that the field of view are different for the Sharp inlet edge, which are $3.47 \mathrm{~mm} \times 3.47 \mathrm{~mm}$ and $3.00 \mathrm{~mm} \times 3.55 \mathrm{~mm}$ for the front and side view respectively. Considering the highest velocity measured in the spray of the order of $200 \mathrm{~m} / \mathrm{s}$, the blurring effect remains about 1 pixel with this optical setup, which has a marginal impact on the image quality [24].

The system operates at low frequency: one injection occurs every second and one image-pair is acquired during one injection cycle. In the reference frame used to give the positions in the spray, the origin $O$ is fixed at the geometric centre of the orifice (see Figure 1), $Y$ represents the axial injection direction and $X$ represents the transverse direction. Image-pairs are used to compute velocity fields in the spray from the displacement of the liquid structures and ligaments in the images. The instantaneous velocities are denoted as $U_{n}$ and $V_{n}$ along $X$ and $Y$ directions,
\end{abstract}




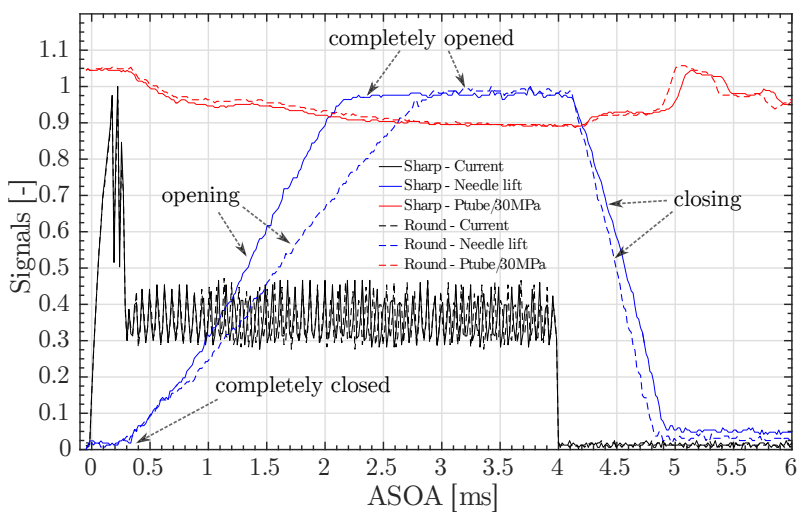

Figure 2. Analog signals vs delay After Start of Activation (ASOA). The current and needle lift signals are rescaled. The pressure signal upstream the injector $\left(P_{\text {tube }}\right)$ is rescaled by the value of $30 \mathrm{MPa}$.

respectively.

Displacements are estimated by liquid interface tracking algorithm. By using the in-house code, the cross-correlation technique is applied to liquid elements (ligaments and droplets) identified in the image. For the sake of brevity, the method is not described here and details can be found in $[19,20]$. Over 250 velocity fields are considered in a statistical analysis to estimate the average and fluctuating velocity in the spray at a given delay $A S O A$.

The spray dynamics is investigated at fully opened stage. The average spray image is shown in figure 3 in gray scale in the background. Liquid elements appear as black over white (for the surrounding air) as commonly obtained with backlight techniques. The mean spray images seem to be quite similar in both cases for the front view, with a narrower spray span for the Round case. More precisely, at a distance of 5D from the orifice, the width is about $30 \%$ less.

The average velocity along $X$ direction over $N$ samples is computed as $\bar{U}=\int_{n} U_{n} / N$. Reynolds decomposition gives the fluctuation term: $u^{\prime}=U-\bar{U}$ and then the variance is computed as $\frac{n}{u^{\prime} u^{\prime}}$, which is the square of standard deviation (std). The average velocity maps in color scale overlap the average spray images in figure 3 . The statistical convergence for Round edge is investigated at two locations in the side view: on the left side (1) and on the right side (2). These locations are represented in figure 3 (lower left) and their coordinates are given in Table 1.

The curves in Figure $4 \mathrm{a}$ indicates the variations of the statistical quantities in both directions when increasing the number of samples. At any location, values are normalised by the value computed with all available samples $(N=250)$, in order to highlight the trends of the curves. Here, filled symbols indicate the values within an interval of $\pm 5 \%$. It is clear that average velocities converge quickly as about 130 samples are sufficient in both directions to reach the final value. For the variances, 200 samples are necessary to obtain the convergence. The statistics for the entire sample set are given in Table 1. The standard deviation is of the same order of magnitude for each side: about $22 \mathrm{~m} / \mathrm{s}$ in the spray direction and $6.5 \mathrm{~m} / \mathrm{s}$ in the transverse direction. Finally, we estimate the precision of the velocity measurement by computing the confidence interval. For uncorrelated samples where $\mathrm{N}>30$, the true mean falls within $95 \%$ of the confidence interval given by $\bar{U} \pm 1.96 \sqrt{\overline{\overline{u^{\prime} u^{\prime}}}} / \sqrt{N}$ [25]. With the values in Table 1, confidence intervals are about $\pm 3 \mathrm{~m} / \mathrm{s}$ and $\pm 0.8 \mathrm{~m} / \mathrm{s}$ for $\bar{U}$ and $\bar{V}$ in $\mathrm{x}$ and y directions respectively. So the estimation of average velocity is accurate within less than $3 \%$ by using this measurement method.

For the Round case, the order of magnitude of the maximum velocity is about $150 \mathrm{~m} / \mathrm{s}$, which is about $10 \%$ less than for the Sharp case. The velocity map on the front view seems to be symmetric for the Sharp edge whereas it is not for the Round edge. Also, velocity values on the side view are significantly reduced on the right side of the spray. It is obvious that the change of radius of curvature at the inlet edge has a huge impact on the spray dynamics from both views. It is most likely a direct consequence of the modification of the flow upstream the orifice, cavitation inception being sensitive to the sac geometry. To better understand the leading reason at the origin of such a difference in the velocity fields for the two nozzle geometries, we focused our attention to the transient phase, i.e., during the lifting of the needle, in the following sections.

\section{Transient phases of injection}

Image-pairs were recorded for different delays $A S O A$, giving average velocity maps along injection duration. The time evolution of the average velocity is considered hereafter (see Figure 5). Let's first consider the front view. The average velocity is computed as follows. Two rectangular regions are considered for computing mean velocities $(5 \mathrm{x}$ 5 pixels) (see gray rectangles in Figure 3). They are located within $X=1.25 D$ on either side of the orifice axis and at a distance of $Y=5 D$ downstream the orifice. According to the needle dynamics for the two cases, the injection duration is about $2.5 \mathrm{~ms}$ and $3.5 \mathrm{~ms}$ for the Sharp and Round cases respectively.

For the Sharp edge a steep increase of the velocity is observed at the early stage of the injection (up to a delay $A S O A \simeq 1 \mathrm{~ms}$ ). The curves have similar shapes and values for both sides, as a result of the symmetry of the spray in this case. A short plateau of $\simeq 0.5 \mathrm{~ms}$ in duration followed by a decrease is observed on the left side of the spray whereas the plateau remains up to the end of the curve on the right side. 

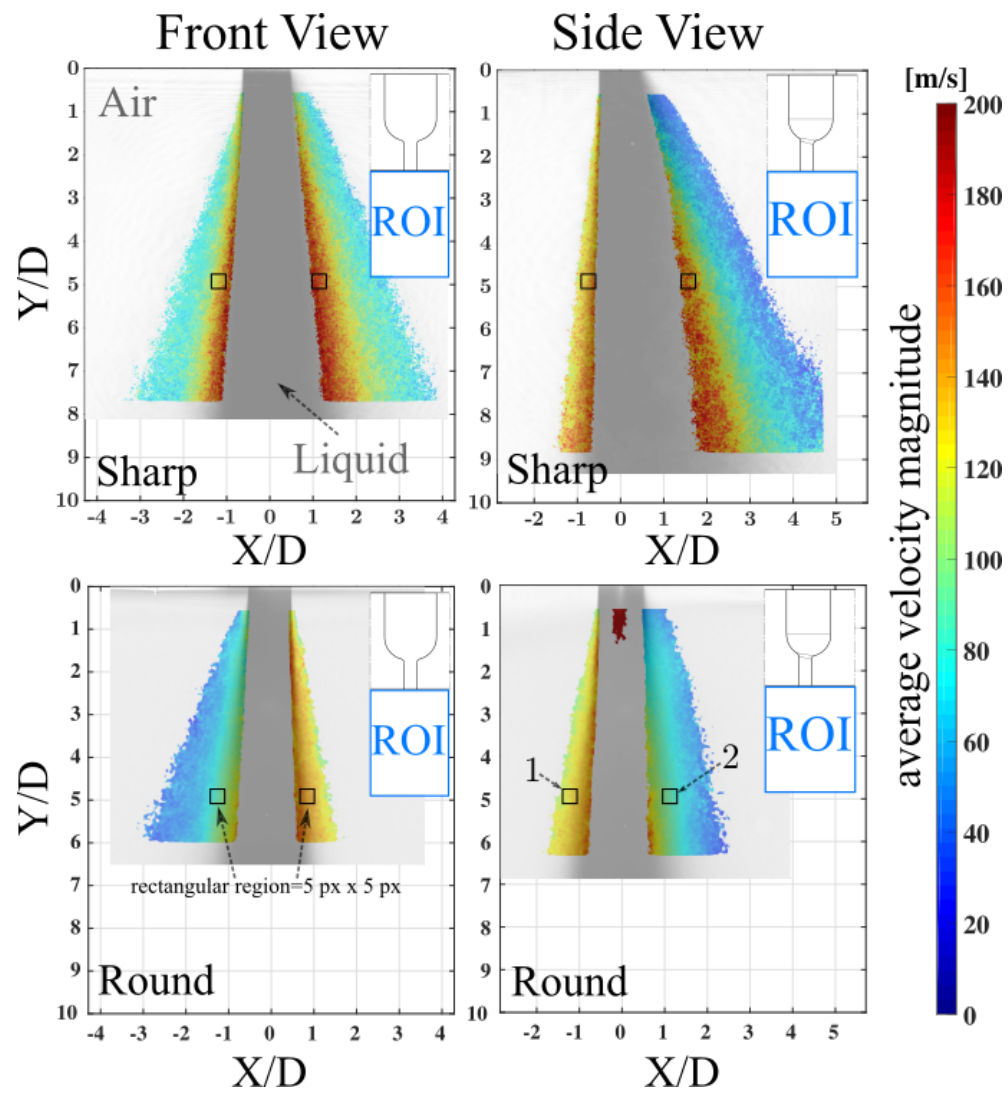

Figure 3. Average velocity map at fully opened stage. The average velocity (in color scale) and the average spray image (in the background) is overlapped. Two upper images, obtained in [21], concern the Sharp inlet edge at $A S O A=2.2$ ms with a spatial resolution of $590 \mathrm{pixel} / \mathrm{mm}$. And the image-pairs are separated by $d t=314 \mathrm{~ns}$; Two lower images concern the Round inlet edge at $A S O A=3 \mathrm{~ms}$ with a spatial resolution of $759 \mathrm{pixel} / \mathrm{mm}$ and $d t=200 \mathrm{~ns}$.

This leads to a non-negligible difference of about $40 \mathrm{~m} / \mathrm{s}$ between the two sides of the spray. A pressure oscillation in the sac induced by a needle rebound when the needle reaches complete lift position could be the reason for this asymmetry. This pressure oscillation could also be induced by pressure wave reflections in the high pressure line, even if the delay for such reflections is sought to be longer in practice (see for example $P_{t u b e}$ signals in Figure 2).

\begin{tabular}{rcc}
\hline Location & 1 & 2 \\
\hline$(x / D, y / D)$ & $(-1.25 ; 5)$ & $(1.25 ; 5)$ \\
$\bar{U}[\mathrm{~m} / \mathrm{s}]$ & 126,6 & 89,0 \\
$\sqrt{\overline{u^{\prime} u^{\prime}}}[\mathrm{m} / \mathrm{s}]$ & 21.3 & 22.1 \\
$\bar{V}[\mathrm{~m} / \mathrm{s}]$ & -21.6 & 13 \\
$\sqrt{\overline{v^{\prime} v^{\prime}}}[\mathrm{m} / \mathrm{s}]$ & 6.5 & 6.5 \\
\hline
\end{tabular}

Table 1. Statistics of velocity in the Round case.

Despite this unexpected outcome, average velocity and needle lift curves have common trends for the Sharp case. For the Round edge, the velocity increase at the beginning of injection is slower than for the Sharp edge. This is expected since the relatively slow needle lift motion for the Round edge indicates a slower pressure increase in the sac in this case. The average velocity curves have similar shapes but reach different values on each side of the spray for the Round edge, with a difference that increases up to $30 \mathrm{~m} / \mathrm{s}$ (starting from a delay $A S O A=1 \mathrm{~ms}$ ). Whereas nozzle geometries are supposed to be symmetrical for this view, it is guessed that a very little asymmetry can subsists in the nozzle geometry for this view. In particular, a slight turn of the PMMA plate could happened during the machining of transparent nozzles. Thus, as cavitation development is very sensitive to the geometry, such a small non visible asymmetry can be the cause for this non expected asymmetrical development of the spray. The right figure in 5 shows the time evolution of velocity in the side view. For the Sharp geometry, it should be mentioned that the rectangular regions are changed to new locations: $X=-0.8 D$ and $X=1.7 D$ on each side of the spray with the same distance to the orifice $(5 \mathrm{D})$, due to the asymmetric spreading of the spray. The shape of curves are not much different for each side, neither from those in the front view whereas the values differs during the transient phase. For Round shape, it should be noticed that the locations of rectangular regions are unchanged. For both sides, the velocities are of the same order as the Sharp edge at the early stage. The same shape is found for each side of the jet, but with a difference about $40 \mathrm{~m} / \mathrm{s}$ between left and right velocities when the plateau is 

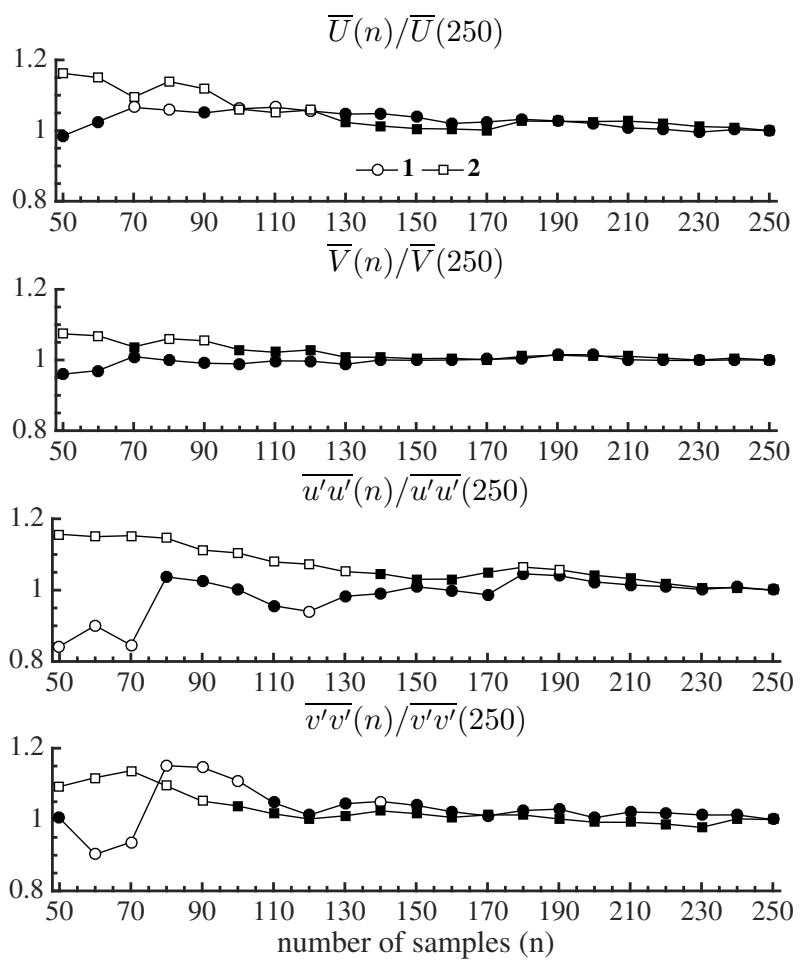

Figure 4. Statistical convergence in the Round case. Filled symbols indicate the values within $[0.95 ; 1.05]$ interval.

reached. This difference is expected in this case.

It is interesting to notice that the difference between each side appears around $A O S A=1 \mathrm{~ms}$. And this seems to be amplified during the transient phase and ends at a value of about $40 \mathrm{~m} / \mathrm{s}$. To develop a better understanding of this results, three instantaneous images of the flow inside the Round edge nozzle for $A S O A=1 \mathrm{~ms}$ are shown in Figure 5). We recall that the aim of asymmetrical configuration is to obtain an asymmetrical development of cavitation allowing a better visualization of the cavities and liquid-vapour interfaces. On these images, the liquid phase or the transparent nozzle appear in light gray, and the vapour cavities induced by cavitation appears in black. It is obvious that cavitation occurs at nozzle entrance on the right side in all images but not always down to the nozzle orifice. We can remark also that large vapour pockets reach the nozzle orifice on the left side of the image. As the presence of cavitation reduce the hydraulic section, the liquid velocity is locally increased in region where there is no cavitation pockets, i.e., mainly on the right side of the flow. In consequence, it is not surprising to obtain larger velocity on the right side than the left side when cavitation is sufficiently developed for the vapour pockets to reach the orifice. Fluctuation levels (std) estimated for the Round edge to be larger than $10 \mathrm{~m} / \mathrm{s}$ are though to be linked to the statistical variation of the properties of the vapour pockets. Indeed, the nozzle orifice is filled with vapour cavities: partially on left and right sides in image No 31; almost completely in image No 32; and mainly on the left side in image No 33. Again, during the development of the spray, the fluctuation level is doubled: almost $25 \mathrm{~m} / \mathrm{s}$ of fluctuation is found at fully opened stage. The fluctuation of the vapour cavities reaching the orifice might be one of the main reasons of this high fluctuation level of the velocity in the near field of the spray.

In the next section, attention is put on the generation of cavitation in the nozzle along the injection duration.

\section{Cavitation analysis}

To investigate the cavitation occurring in the nozzle, backlight images of the flow in the nozzle are recorded (for the side view only) with a similar optical set-up. It is necessary to be pointed out that as for any imaging method, no data can be provided for the size and shape of the vapour pockets in the dimension perpendicular to the image plane due to the 2D projection proceeded by image formation. Thus, a low gray level pixel in the image indicates that vapour phase is present in the nozzle at the corresponding $(X, Y)$ position, but without any indication on the position in the third direction nor on the depth of the local the vapour phase pocket. These images thus need to be interpreted with caution. Whereas the volume of cavitation pockets cannot be determined, the relative frequency of the occurrence of cavitation was estimated by measuring the relative size of the projection of these pockets on the images.

The method to estimate the relative frequency of cavitation is demonstrated through an example in the figure 6 . The gray level raw image is first transformed in a two-level (black and white, bw) image by applying a threshold value determined by the Ostu's threshold method [26] on an average image at fully opened stage. The bw image is then inverted, in order to have white pixels (coded with value 1) corresponding to the presence of the vapour phase. Finally, the average image is computed over 25 samples at the same delay $A S O A$. This average image has a null gray level value, i.e. null relative frequency in region where cavitation never occurred and a gray level of 1 , i.e. a 


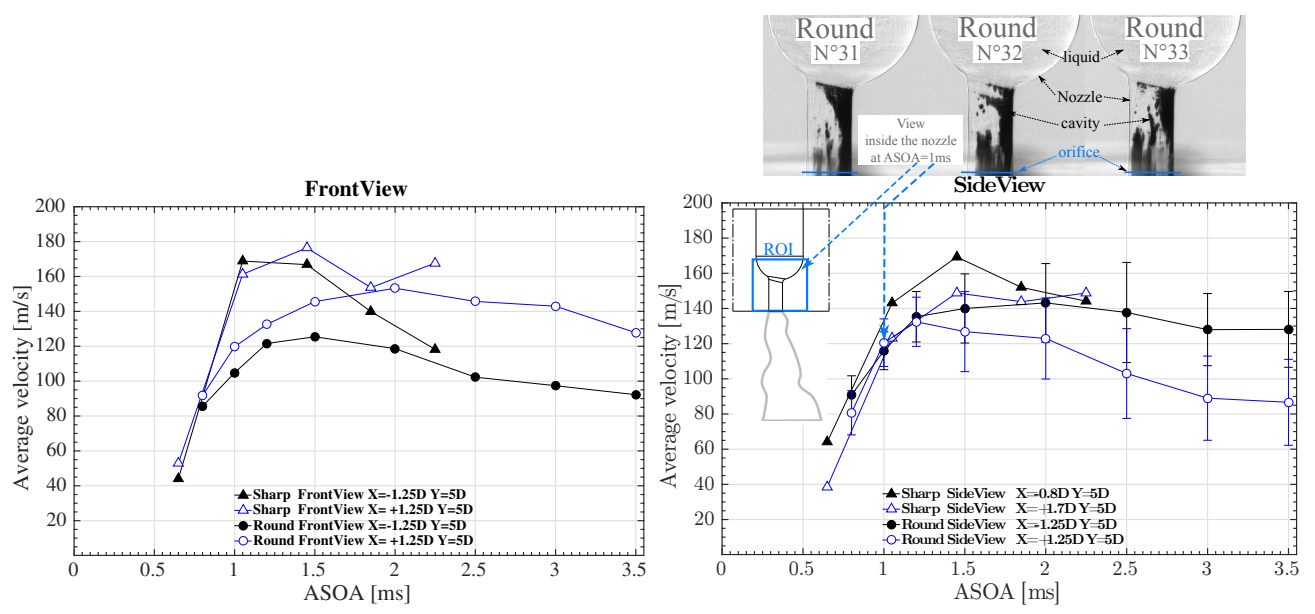

Figure 5. Time evolution of the average velocity from the front (left) and side (right) view.
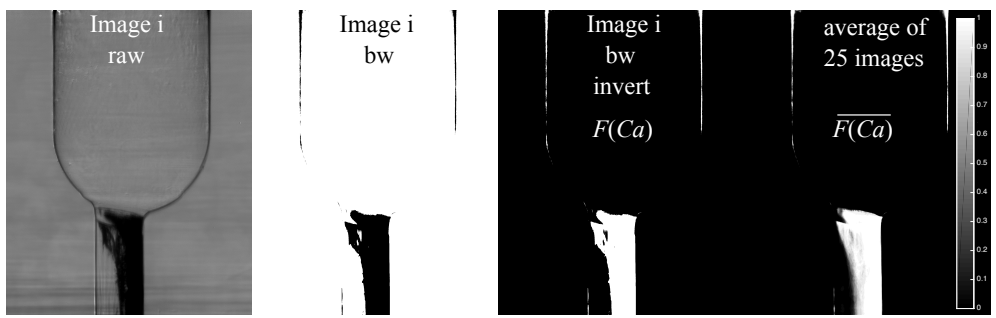

Figure 6. Computation of the average relative frequency of cavitation.

relative frequency of 1 , where cavitation is always detected in the image series.

The average images giving the cavitation frequency $\overline{F(C a)}$ at fully opened stage for both geometries are shown on the right side of the figure 7 . Despite the similar shape of the light gray area in the region of interest, the gray level is clearly different for the different geometries. The variation of the frequency along the nozzle axis was determined by averaging the gray level values over each row in the image. The reference position of these row are given in distance over $Y$ axis from the nozzle orifice, i.e., with negative values.

The evolution of the frequency $\overline{F(C a)}$ at the entrance and exit of the orifice channel is first examined. At the entrance $(Y=-0.75 \mathrm{~mm})$, the increase of the frequency $\overline{F(C a)}$ is fast for the Sharp inlet edge, and the plateau is reached at a very early stage, before $1 \mathrm{~ms}$. The increase is also very steep for Round edge but cavitation occurrence starts with a delay of $\simeq 0.1 \mathrm{~ms}$ and followed later on by a plateau. This means that cavitation is initiated in a very short time at orifice entrance whatever the velocity of the needle lift motion. Moreover, for the Sharp edge, the plateau value over 0.9 indicates that for any injection event, cavitation is mostly present at the entrance of the orifice.

At the orifice outlet $(Y=-0.05 \mathrm{~mm})$, the cavitation frequency starts increasing up to a delay $A S O A \simeq 1 \mathrm{~ms}$ and then decreases slowly. When the needle is lifted completely, we find that the width of the white region is approximately half the orifice diameter (see image in Figure 7), which is consistent with the value of $\overline{F(C a)} \simeq 0.5$. It can be noticed that the curves for the frequency and for the average velocity look very similar for the Sharp edge (see Figure 5). Indeed, the fast increase of the near-field velocity occurs at the same time (for $A S O A<1 \mathrm{~ms}$ ) and the same way as for the start of the cavitation frequency.

We can also notice that for the Round edge cavitation starts nearly simultaneously at the entrance and at the exit of the orifice, with a delay of about $0.2 \mathrm{~ms}$ compared to the Sharp edge. At the entrance, the frequency continue to increase up to a plateau of about 0.7 , which is $30 \%$ lower than for the Sharp edge, showing that cavitation is less prompt to occur for a smooth shaped orifice inlet. At the nozzle exit, the frequency curves are very similar for both shapes, i.e., it decreases to a value of $\overline{F(C a)} \simeq 0.5$ meaning that cavitation occurred here about half of the time. In addition, a slow transition along with fluctuations are both encountered in the time variation of velocity and cavitation frequency curves. It is reasonable to suggest that the development of cavitation at nozzle exit is very sensitive to the inlet edge geometry, and the spray atomization in the near-field is related to the way cavitation is developing up to the nozzle exit.

The variation of cavitation frequency along the orifice at any time after the start of injection is shown in the figure 8 . For the Sharp edge, the profiles of $\overline{F(C a)}$ are quite similar during the injection and present a quasi-continuous decrease of cavitation frequency from inlet to outlet. For the Round edge, the variation of cavitation present a minimum at mid distance from $0.7 \mathrm{~ms}$ to $2.0 \mathrm{~ms}$. Cavitation frequency becomes continuously decreasing only for a delay $A S O A \geq 2.5 \mathrm{~ms}$.

At fully opened stage, cavitation frequency reaches similar values for both nozzles, which is not consistent with the difference observed previously on the velocity maps (see Figure 3). As mentioned earlier, images do not contain 


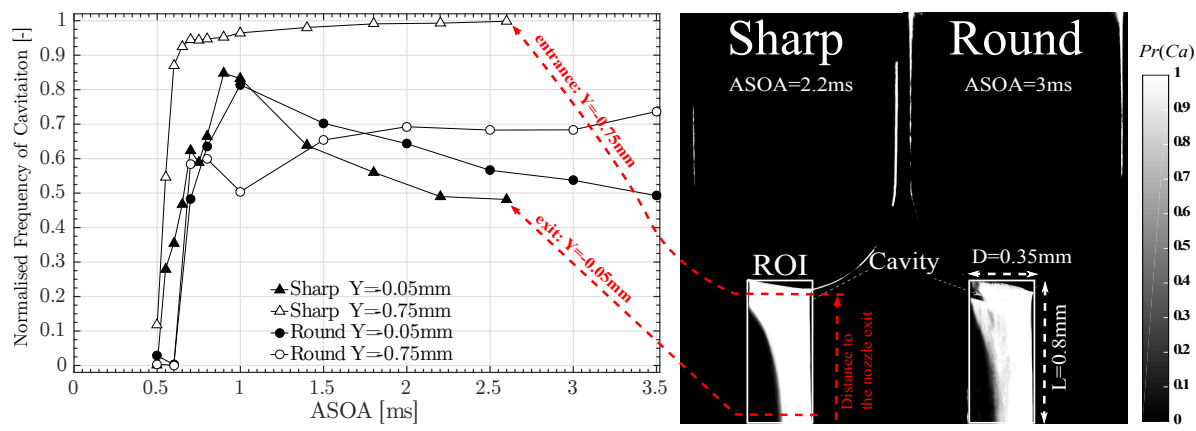

Figure 7. Average relative frequency of cavitation $\overline{F(C a)}$ at the entrance $(Y=-0.75 \mathrm{~mm})$ and the exit $(Y=-0.05 \mathrm{~mm})$ of the nozzle orifice.
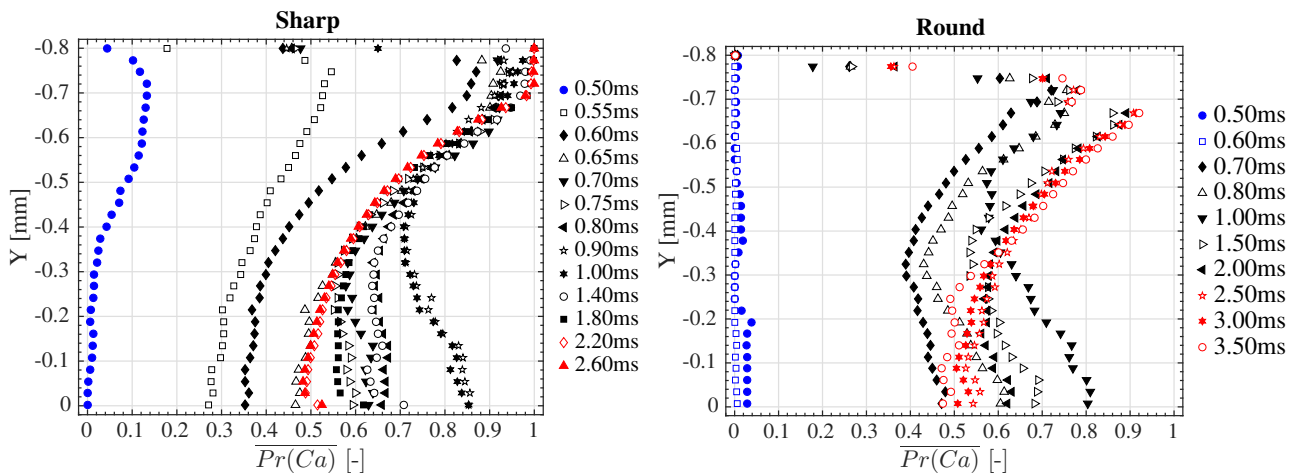

Figure 8. Average relative frequency of cavitation $\overline{F(C a)}$ profiles for the Sharp case (left) and Round case (right).

any information on the third dimension. Two vapour pockets having the same projection area in image can have totally different depths in the third dimension. These pockets leads to similar cavitation frequency with the above mentioned analysis, but the spray dynamics will probably be different. Thus, the missing information in the third dimension is believed to be a key element to well understand the effect of cavitation on atomization.

\section{Conclusions}

This experimental work investigates the flow through a diesel-like transparent nozzle. Two nozzles with different inlet edge shapes were compared. A backlight technique was used to visualize the flow downstream the orifice within ten orifice diameters and the flow in the nozzle.

Velocities of the spray estimated by a cross-correlation method were statistically averaged. During the transient phase, the velocity variations were compared to report the high sensitivity of the flow pattern to the orifice shape. This yields to different velocity maps at fully opened stage. The relative frequency of cavitation in the nozzle were computed by measuring the relative size of the vapour pockets on the image. For the Sharp edge, cavitation occurs immediately and a continuous decrease of the frequency of the cavitation is observed after $A S O A=1 \mathrm{~ms}$ and from inlet to outlet. For the Round edge, cavitation occurs with a short delay compared to the Sharp edge, and the cavitation frequency varies gradually after $A S O A=1 \mathrm{~ms}$ and present a minimum value at mid nozzle distance. The spatial distribution of cavitation frequency from inlet to outlet of the orifice is thus found to be different between the two nozzles. The results indicate that the velocity in the spray and the frequency of cavitation in the nozzle have similar trends for both nozzle designs.

Perspectives for this work include improvement of the determination of the properties of vapour pockets in the nozzle. This would imply a measure of the depth of the vapour pockets in the third dimension. Due to the complex 3D geometry of the small-size nozzle, this will constitute a real challenge to overcome, mostly from an optical point of view. In a more accessible way, the temporal correlation of the flow inside the nozzle and downstream the orifice will have to be deeply explored by simultaneous visualization of inside and outside flows.

\section{Acknowledgements}

This work was supported by CANNEx program (ANR-13-TDMO-03), funded from French National Research Agency (ANR). 


\section{References}

[1] Lefebvre, A., 1988. Atomization and sprays, Vol. 1040. CRC press.

[2] Nurick, W., 1976. "Orifice cavitation and its effect on spray mixing". ASME, Transactions, Series I-Journal of Fluids Engineering, 98, pp. 681-687.

[3] Winklhofer, A. H. A. M. E., and Phillip, H., 2000. "Cavitation and spray formations in diesel flow situations". In ILASS-Europe, pp. 11-13.

[4] Winklhofer, E., Kull, E., Kelz, E., and Morozov, A., 2001. "Comprehensive hydraulic and flow field documentation in model throttle experiments under cavitation conditions". In ILASS-Europe, pp. 2-6.

[5] Sou, A., Hosokawa, S., and Tomiyama, A., 2007. "Effects of cavitation in a nozzle on liquid jet atomization". International journal of heat and mass transfer, 50(17), pp. 3575-3582.

[6] Prasetya, R., Mashida, M., Yamada, Y., and Sou, A., 2015. "Effect of nozzle inlet roundness and nozzle length on cavitation and liquid jet". In ILASS-Europe.

[7] Pratama, R. H., Sou, A., Wada, Y., and Yohohata, H., 2015. "Cavitation in mini-sac nozzle and injected liquid jet". In ILASS-Europe.

[8] Dabiri, S., Sirignano, W., and Joseph, D., 2007. "Cavitation in an orifice flow". Physics of Fluids, 19(7), p. 072112.

[9] Bastawissi, H. A.-E., Elkelawy, M., et al., 2014. "Investigation of the flow pattern inside a diesel engine injection nozzle to determine the relationship between various flow parameters and the occurrence of cavitation". Engineering, 6(13), p. 923.

[10] Payri, F., Bermudez, V., Payri, R., and Salvador, F., 2004. "The influence of cavitation on the internal flow and the spray characteristics in diesel injection nozzles". Fuel, 83(4), pp. 419-431.

[11] Payri, R., García, J., Salvador, F., and Gimeno, J., 2005. "Using spray momentum flux measurements to understand the influence of diesel nozzle geometry on spray characteristics". Fuel, 84(5), pp. $551-561$.

[12] Payri, R., Salvador, F. J., Gimeno, J., and Viera, J. P., 2015. "Experimental analysis on the influence of nozzle geometry over the dispersion of liquid n-dodecane sprays". Frontiers in Mechanical Engineering, 1, p. 13.

[13] Wu, Z., Gao, Y., Gong, H., and Li, L., 2016. "Evaluation and optimization of the structural parameter of diesel nozzle basing on synchrotron radiation imaging techniques". Journal of Instrumentation, 11(04), p. C04004.

[14] Sou, A., Tomiyama, A., Hosokawa, S., Nigorikawa, S., and Maeda, T., 2006. "Cavitation in a two-dimensional nozzle and liquid jet atomization". JSME International Journal Series B Fluids and Thermal Engineering, 49(4), pp. 1253-1259.

[15] Mauger, C., Méès, L., Michard, M., Azouzi, A., and Valette, S., 2012. "Shadowgraph, schlieren and interferometry in a $2 \mathrm{~d}$ cavitating channel flow". Exp. Fluids.

[16] Hult, J., Simmank, P., Matlok, S., Mayer, S., Falgout, Z., and Linne, M., 2016. "Interior flow and near-nozzle spray development in a marine-engine diesel fuel injector". Experiments in Fluids, 57(4), pp. 1-19.

[17] Suh, H. K., and Lee, C. S., 2008. "Effect of cavitation in nozzle orifice on the diesel fuel atomization characteristics". International journal of heat and fluid flow, 29(4), pp. 1001-1009.

[18] Moon, S., Gao, Y., Wang, J., Fezzaa, K., and Tsujimura, T., 2014. "Near-field dynamics of high-speed diesel sprays: Effects of orifice inlet geometry and injection pressure". Fuel, 133, pp. 299-309.

[19] Sedarsky, D., Idlahcen, S., Rozé, C., and Blaisot, J.-B., 2013. "Velocity measurements in the near field of a diesel fuel injector by ultrafast imagery". Experiments in fluids, 54(2), pp. 1-12.

[20] Berlemont, A., Blaisot, J.-B., Bouali, Z., Cousin, J., Desjonqueres, P., Doring, M., Dumouchel, C., Idlahcen, S., Leboucher, N., Lounnaci, K., et al., 2013. "Numerical simulation of primary atomization: Interaction with experimental analysis". Atomization Sprays, 23(12), pp. 1103-1138.

[21] Purwar, H., Lounnaci, K., Idlahcen, S., Roze, C., Blaisot, J.-B., Méès, L., and Michard, M., Aug. 23.-27. 2015. "Effect of cavitation on velocity in the near-field of a diesel nozzle". In 13th Triennial International Conference on Liquid Atomization and Spray Systems.

[22] Chorążewski, M., Dergal, F., Sawaya, T., Mokbel, I., Grolier, J.-P. E., and Jose, J., 2013. "Thermophysical properties of normafluid (iso 4113) over wide pressure and temperature ranges". Fuel, 105, pp. 440-450.

[23] Desantes, J., Payri, R., Salvador, F., and De la Morena, J., 2010. "Influence of cavitation phenomenon on primary break-up and spray behavior at stationary conditions". Fuel, 89(10), pp. 3033-3041.

[24] Purwar, H., Wang, H., Tang, M., Idlahcen, S., Rozé, C., Blaisot, J.-B., Godin, T., and Hideur, A., 2015. "Ultrafast high-repetition imaging of fuel sprays using picosecond fiber laser". Optics express, 23(26), pp. 33396-33407.

[25] Benedict, L., and Gould, R., 1996. "Towards better uncertainty estimates for turbulence statistics". Experiments in fluids, 22(2), pp. 129-136.

[26] Otsu, N., 1979. "A threshold selection method from gray-level histograms". IEEE transactions on systems, man, and cybernetics, 9(1), pp. 62-66. 